\title{
Evaluation of Safety and Cost of an Open-Design Oxygen Mask in a Large Community Hospital
}

\author{
Patricia A DeJuilio MSc RRT RRT-ACCS RRT-NPS, Maya B Jenkins MSc RRT, and \\ Jeffrey P Huml MD
}

\begin{abstract}
BACKGROUND: In our large community hospital, we observed that traditional oxygen masks were sometimes set at an inappropriately low flow. We hoped to eliminate this safety concern through adoption of an open-design oxygen mask. We also hoped that more immediate flow changes would lead to a decrease in medical gas consumption. Finally, by standardizing to one mask, goals were to reduce the cost of oxygen delivery. METHODS: We conducted a retrospective analysis, 12 months before and 12 months after implementation of the open-design oxygen mask. Unusual occurrence reports related to supplemental oxygen delivery were reviewed. Oxygen device use and bulk oxygen consumption were recorded. The total number of patient days was obtained from the electronic medical record. RESULTS: There were no unusual occurrence reports or concerns involving an oxygen device in those areas that converted to the open-design oxygen mask. In fiscal year 2014 , bulk oxygen use was $13,036,686$ cubic feet, and there were 74,734 patient days. In fiscal year 2016, bulk oxygen use was $12,072,610$ cubic feet and there were 99,428 patient days. The reduction in oxygen consumption was $\mathbf{\$ 3 , 6 7 0}$ despite the increase in patient days. In fiscal year $\mathbf{2 0 1 4}$, 3,848 oxygen devices were used for a cost of $\$ 3,411$, and in fiscal year 2016, 5,512 devices were used for a cost of $\$ 12,963$. The net savings from open-design oxygen mask conversion was $\$ 23,487$ annual and corrected for increased patient population. Oxygen consumption and supply cost per patient day resulted in $\$ 1.19$ per patient day pre-implementation and $\$ 0.95$ after implementation of the open-design oxygen mask $(P=.003)$. CONCLUSIONS: The open-design oxygen mask may be a safe and less costly alternative to traditional oxygen delivery devices. Key words: oxygen; carbon dioxide; safety; respiration; costs and cost analysis; pulmonary circulation. [Respir Care 2018;63(4):412-416. (c) 2018 Daedalus Enterprises]
\end{abstract}

\section{Introduction}

Several supplemental oxygen delivery devices are commercially available. The clinician must decide which type of device to use based on clinical assessment. ${ }^{1}$ It is not unusual to change the device due to issues of comfort or changing oxygen requirements. At our hospital, we expe-

The authors are affiliated with Northwestern Medicine Central DuPage Hospital, Winfield, Illinois.

The authors have disclosed no conflicts of interest.

Correspondence: Patricia A DeJuilio MSc RRT RRT-ACCS RRT-NPS, Northwestern Medicine Central DuPage Hospital, 25 North Winfield Road, Winfield, IL 60190. E-mail: patricia.dejuilio@nm.org.

DOI: $10.4187 /$ respcare. 05567 rienced unusual occurrence reports related to inadequate flow delivery via closed-mask systems. The open-design oxygen mask can be used at a flow of $1-15 \mathrm{~L} / \mathrm{min}$ to deliver $24-90 \%$ oxygen. ${ }^{2-4}$ Lamb and Piper $^{2}$ reported that $\mathrm{CO}_{2}$ was cleared efficiently with this design. The aim of this study was to determine whether use of an open-design oxygen mask would eliminate the safety concerns we had regarding the risk of $\mathrm{CO}_{2}$ rebreathing in a less costly manner.

\section{Methods}

Study Site

Northwestern Medicine Central DuPage Hospital is a 395-bed acute-care full-service tertiary hospital. During the time period when data were collected, there were no 
major changes in hospital designations or populations served, although the overall census increased. Our hospital has an oxygen protocol that allows nurses and respiratory therapists to titrate oxygen as appropriate to maintain adequate oxygen saturations. There were no changes in policies, nor were there revisions other than change of oxygen devices with implementation of the open-design oxygen mask to system oxygen protocol. Northwestern Memorial Healthcare West Region Research Support determined that this study was exempt from institutional review board review because it was process improvement.

\section{Study Variables}

Data were recorded from July 2013 to June 2014 (fiscal year 2014) and from July 2015 to June 2016 (fiscal year 2016). For fiscal year 2014, traditional oxygen mask devices included simple oxygen masks, adult 3 -in-1 oxygen masks, partial rebreathing masks, non-rebreathing masks, tracheostomy masks, and air-entrainment masks (nasal cannulas continue to be utilized before and after implementation of the open-design oxygen mask). Fiscal year 2015 was a transitional year, as the open-design mask was introduced to all areas of the hospital over a period of several months. In fiscal year 2016, the open oxygen mask and open tracheostomy mask were used exclusively. Bubble humidifiers were also included in cost analysis, although these are rarely used. The materials management department provided oxygen device cost and usage data. Facilities management provided oxygen gas consumption use and cost data. The bulk oxygen consumption comparison used reports provided by the medical supply company to our facilities management department. The cost per square cubic foot of medical oxygen gas increased during the time periods studied (from $\$ 0.00059$ to $\$ 0.0070$ ).

A retrospective study was conducted to evaluate reported safety issues related to oxygen devices, oxygen device use and cost, and oxygen gas consumption at our suburban hospital. The analysis was conducted for a 12month period pre-implementation and a 12-month period post-implementation of the open-design oxygen mask. The total number of patient days for all subjects independent of whether they received supplemental oxygen was obtained from the electronic medical record. Oxygen device use and bulk oxygen utilization data were obtained for the emergency department, medical care units, 2 adult critical care units, pediatric ICU, pediatrics, behavioral health, and neonatal ICU. There were no areas excluded from data collection; however, the emergency department continued to use non-rebreathing masks for most of their patients until December 2015 due to paramedic supply access. Additionally, the labor and delivery unit delayed conversion to the open-design oxygen mask until the fall of 2015.

\section{QUICK LOOK}

\section{Current knowledge}

Supplemental oxygen delivery is routine and necessary to treat patients to maintain adequate tissue oxygenation and to minimize cardiopulmonary work in patients who are hypoxemic, have shortness of breath, or are hemodynamically unstable. The choice of delivery device is complex and may require minimum flows to ensure patient safety. Safety, cost, and efficiency are considerations for most product decisions made by hospitals today, including oxygen delivery devices.

\section{What this paper contributes to our knowledge}

Implementation of an open-design oxygen mask at a large community hospital improved patient safety and significantly reduced cost. We previously had reports of inadequate flows delivered causing a concern for $\mathrm{CO}_{2}$ rebreathing, and since implementation of the opendesign oxygen mask, we have had no concerns. Oxygen consumption and supply cost per patient day were studied, and cost per patient day was significantly reduced.

The case mix index was assessed to determine overall subject complexity in the 2 groups. The case mix index is the average relative weight of Medicare severity-diagnostic-related groups and is typically used to determine staffing and resources required. ${ }^{5}$ We collected all unusual occurrence reports related to supplemental oxygen delivery devices pre- and post-implementation.

\section{Data Analysis}

Descriptive data were collected for patient days and bulk oxygen. Statistical control charts (X-bar and R) were composed to evaluate month to month variation using Microsoft Excel. The total oxygen delivery costs per 1,000 patient days per month were compared with a WilcoxonMann-Whitney test for the 2014 and 2016 fiscal years and tested at $\alpha=.05$. The analysis was performed in Microsoft Excel, and statistical analysis was done using QI-Macros 2017 (Know Ware International, Denver, Colorado).

\section{Results}

There were 3 unusual occurrence reports submitted from July 2103 to June 2014. Two non-rebreather masks were found on subjects at $<5 \mathrm{~L} / \mathrm{min}$, and one simple mask was found at $2 \mathrm{~L} / \mathrm{min}$. In the year before complete conversion to the open-design oxygen mask, there was one additional 


\section{Evaluation of an Open-Design Oxygen Mask}

Table 1. Patient Days, Bulk Oxygen Use, and Number of Oxygen Masks Used During Time Periods Studied

\begin{tabular}{|c|c|c|c|c|}
\hline & July 2013 to June 2014 & June 2015 to July 2016 & Difference: July 2016 - June 2014 & Change, $\%$ \\
\hline Patient days, no. & 74,734 & 99,428 & 24,694 & $33.0 \%$ \\
\hline Bulk oxygen, $\mathrm{ft}^{3}$ & $13,036,686$ & $12,072,610$ & $-964,076$ & $-7.4 \%$ \\
\hline Oxygen cost, USD & $85,472.46$ & $81,802.72$ & $-3,669.74$ & $-4.3 \%$ \\
\hline $\mathrm{O}_{2}$ cost/subject, USD & 1.14 & 0.82 & $(0.32)$ & $-28.1 \%$ \\
\hline \multicolumn{5}{|l|}{ Oxygen masks } \\
\hline Total units, no. & 3,848 & 5,512 & 1,664 & $43.2 \%$ \\
\hline Mask cost, USD & $3,411.11$ & $12,963.27$ & $9,552.16$ & $280.0 \%$ \\
\hline Mask cost/patient, USD & 0.05 & 0.13 & 0.08 & $185.6 \%$ \\
\hline Total cost/patient, USD & 1.19 & 0.95 & $(0.24)$ & $-19.9 \%$ \\
\hline USD $=$ US dollars & & & & \\
\hline
\end{tabular}

report of a non-rebreathing mask at inadequate flow on the labor and delivery unit. From July 2014 to June 2016, there were no unusual occurrence reports or concerns involving an oxygen device in those areas that converted to the open-design oxygen mask, despite the overall increase in the number of safety reports based on internal quality data.

Annual bulk liquid oxygen use decreased with the introduction of the open-design oxygen mask. Bulk oxygen use during fiscal year 2014 was 13,036,686 cubic feet for 74,734 patient days, and during fiscal year 2016, 12,072,610 cubic feet was used for 99,428 patient days (a reduction of 964,076 cubic feet with an increase of 24,694 total patient days). The reduction in oxygen consumption realized $\$ 3,670$ in savings even as the number of patients in the hospital increased by $33 \%$. The number of oxygen devices used during fiscal year 2014 was 3,848, and it was 5,512 during fiscal year 2016. This increase of masks used may reflect the $33 \%$ increase in patient population and increased clinical complexity (case mix index increased from 1.4195 to 1.4806 during the time periods studied). The cost for oxygen devices was $\$ 3,411$ during fiscal year 2014 and $\$ 12,963$ during fiscal year 2016 (Table 1). Oxygen consumption and supply cost per patient day resulted in $\$ 1.19$ per patient day pre-implementation and $\$ 0.95$ per patient day after implementation of the open-design oxygen mask $(P=.003)$ (Fig. 1). Statistical control charts (X-bar and R) evaluated month to month variation and found the variation during both time intervals to be "in control" with means of $\$ 1,190.24$ and $\$ 846.07$ (Fig. 2).

\section{Discussion}

In this study, we experienced a reduction in the number of safety occurrence reports due to device use after introducing this mask design. There were no safety occurrence reports involving the use of the open-design oxygen mask. We also experienced a significant cost reduction with the

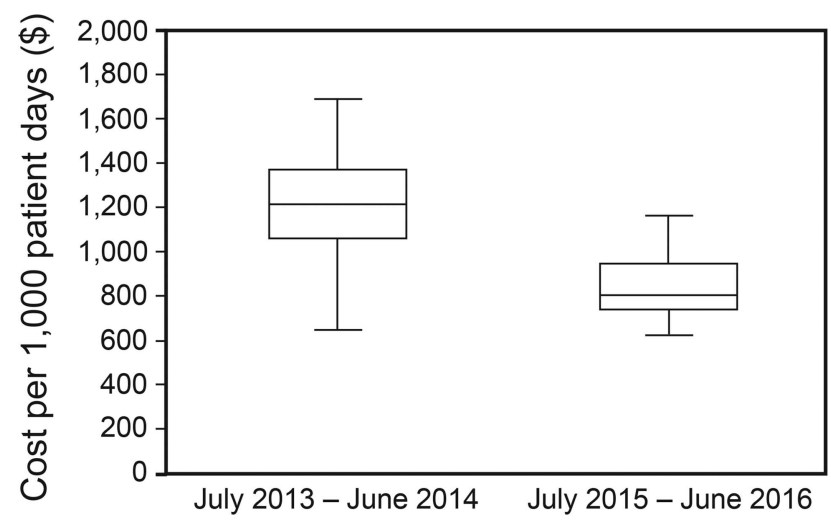

Fig. 1. Supplemental oxygen cost for 2 populations compared using a box and whisker plot. Whiskers indicate maximum and minimum cost. Center lines denote median. The bottom sections of the boxes represent first quartile data, and top sections of the boxes are third quartile data. The difference between the 2 groups is statistically significant $(P=.003)$ by Wilcoxon-Mann-Whitney test.

implementation of the open mask design for oxygen delivery. Although the individual devices are more expensive, cost savings were realized through a reduction in the number of devices used and oxygen consumption.

At our hospital, we use a protocol implemented by respiratory therapists and nurses for oxygen delivery. Such protocols have been shown effective. ${ }^{6-9}$ Despite this protocol, we have observed incorrect use of oxygen delivery devices. A study performed to examine perspectives of nurses on oxygen therapy found that they recognize that the key to effective management of oxygen therapy is their ability to recognize triggers for changing oxygen therapy devices. ${ }^{10}$ By reducing the number of oxygen device choices, it might be easier to use the device correctly, particularly for persons who administer oxygen infrequently. Most devices require specific flows in order to provide safe and effective supplemental oxygen delivery. If flow is too low, the patient can receive a suboptimum 

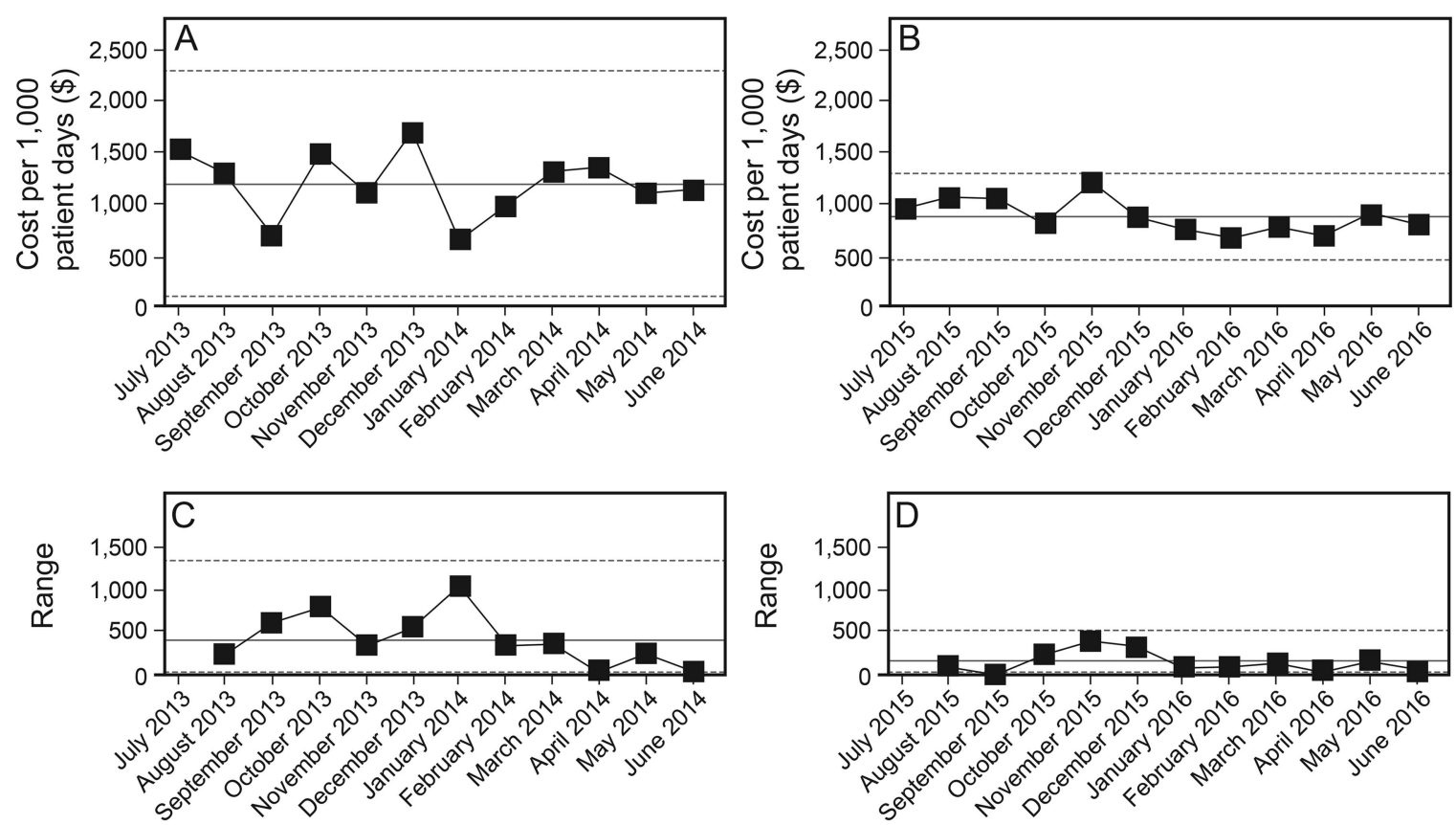

Fig. 2. Statistical control charts (X-bar and R) look for how much variation exists and whether that variation is of an amount that would be predicted with a stable process (ie, in control). In the $\mathrm{X}$ charts ( $\mathrm{A}$ and $\mathrm{B}$ ), the mean is represented by the solid gray line. Dashed gray lines represent upper and lower control limits $( \pm 3 \mathrm{SD}$ ). The process was in control because month-to-month variation never went above or below \pm 3 SD nor violated the other rules of the control chart that would indicate an unstable process. The $R$ charts $(C$ and $D)$ show change in cost that occurred the month previous to the month indicated.

$\mathrm{F}_{\mathrm{IO}_{2}}$. If adequate flow is not maintained with a mask, the patient is in danger of rebreathing exhaled carbon dioxide. One study reported that adequate flow with the non-rebreathing mask adequately cleared $\mathrm{CO}_{2}$, but lower flows cleared less $\mathrm{CO}_{2}{ }^{2}$

We believe that the cost savings reported here were most likely due to the adoption of the open-style mask and not for confounding reasons. The same oxygen protocol was used in both groups. The complexity reflected by the case mix index and the days of oxygen therapy both increased after implementation of the new device. However, as this was an observational study and not a randomized controlled trial, we cannot completely rule out the possibility of these cost savings being attributable to other factors.

Positive patient outcomes in the hospital setting depend largely on the ability of clinicians to administer safe, effective treatments to their patients, and health-care systems are responsible for providing the best tools for clinicians to use. Miller ${ }^{1}$ described the various devices available to clinicians today; however, the open-design oxygen mask is not mentioned in his review, most likely because it is relatively new and understudied. We found that implementing the open-design oxygen mask eliminated the need to change oxygen therapy devices.

Focus on safety is becoming increasingly important, with hospitals developing safety committees, online error- reporting tools, and a focus on high reliability environment and culture of safety. Non-punitive measures and transparency in reporting are common in hospitals to help improve processes, which in turn leads to improved patient safety. As the safety culture in our hospital has improved and continues to improve, there has been an increase in the number of unusual events documenting errors with oxygen delivery. The non-rebreather mask, for instance, can deliver 60-90\% oxygen concentrations with flows from 10 to $15 \mathrm{~L} / \mathrm{min}$ (although high breathing frequencies can significantly reduce the $\mathrm{F}_{\mathrm{IO}_{2}}$ ). ${ }^{1,11,12}$ While using this device, it is important to ensure that the reservoir bag is inflated at least two-thirds full of oxygen. If flow is inadequate, the patient is at risk for rebreathing exhaled carbon dioxide. We had reports of occurrences where the flow was not set adequately to maintain a full reservoir bag, which led to some respiratory distress. Additionally we had a report of a simple mask set at a flow not high enough to eliminate rebreathing risk. This study showed that switching to an open-design oxygen delivery system reduces safety concerns and adheres to financial stewardship. The open-design oxygen mask achieves the goals of systems working to provide value-based medicine to their patients.

There were limitations to this work. This was an observational study, not a randomized controlled trial. We relied on safety event reporting as a means of determining issues specific to types of oxygen masks. Safety events are 


\section{Evaluation of an Open-Design Oxygen Mask}

typically underreported, and although we experienced an overall increase in safety event reports during the time period of this study, it is not possible to determine that these data are reliable. We did not evaluate the appropriateness or the effectiveness of oxygen therapy, nor did we evaluate patient outcomes. Further studies are needed to compare patient outcomes with the use of an open mask. Finally, this is a single-center study and, as such, our results may or may not apply to other settings.

\section{Conclusions}

We found that the open oxygen mask design may be a safe and less costly alternative to traditional oxygen delivery devices.

\section{ACKNOWLEDGMENTS}

We thank Mary Lou Loerzel for providing product usage and cost, Christine Dupplar for providing gas consumption spreadsheets, and Dr Robert Chase for statistical support.

\section{REFERENCES}

1. Miller K. Oxygen administration: what is the best choice? RT for decision makers in Respiratory Care; October 2015. http://www. rtmagazine.com/2015/10/oxygen-administration-best-choice/. Accessed November 27, 2017.
2. Lamb K, Piper D. Southmedic Oxymask compared with the Hudson RCI non-rebreather mask: safety and performance comparison. Can J Respir Ther 2016;52(1):13-15.

3. Paul JE, Hangan H, Haigato J. The OxyMask ${ }^{\mathrm{TM}}$ development and performance in healthy volunteers. Med Devices (Auckl) 2009; 2:9-17.

4. Beecroft JM, Hanly PJ. Comparison of the OxyMask and Venturi mask in the delivery of supplemental oxygen: pilot study in oxygendependent patients. Can Respir J 2006;13(5):247-252.

5. Centers for Medicare and Medicaid Services. Case Mix Index; https://www.cms.gov/Medicare/Medicare-Fee-for-Service-Payment/ AcuteInpatientPPS/Acute-Inpatient-Files-for-Download-Items/ CMS022630.html. Accessed July 20, 2017.

6. Konschak MR, Binder A, Binder RE. Oxygen therapy utilization in a community hospital: use of a protocol to improve oxygen administration and preserve resources. Respir Care 1999;44(5):506-511.

7. Cousins JL, Wark PA, McDonald VM. Acute oxygen therapy: a review of prescribing and delivery practices. Int J Chron Obstruct Pulmon Dis 2016;11:1067-1075.

8. Lodato RF. Oxygen toxicity. Crit Care Clin 1990;6(3):749-765.

9. Fulmer JD, Snider GL. ACCP-NHLBI National Conference on Oxygen Therapy. Chest 1984;86(2):234-247. Concurrent publication in Respir Care 1984;29(9):922-935.

10. Eastwood GM, O'Connell B, Gardner A, Considine J. Patients' and nurses' perspectives on oxygen therapy: a qualitative study. J Adv Nurs 2009;65(3):634-641.

11. Wagstaff TA, Soni N. Performance of six types of oxygen delivery devices at varying respiratory rates. Anaesthesia 2007;62(5): 492-503.

12. Kallstrom T. AARC clinical practice guideline: oxygen therapy for adults in the acute care facility-2002 revision and update. Respir Care 2002;47(6):717-720. 